\title{
ON THE MACROSCOPIC LIMIT OF BROWNIAN PARTICLES WITH LOCAL INTERACTION
}

\author{
FRANCO FLANDOLI \\ Email: franco.flandoli@sns.it. \\ Scuola Normale Superiore, Piazza dei Cavalieri, 7, 56126 Pisa, Italy. \\ MARTA LEOCATA \\ Email: leocata@math.univ-lyon1.fr. \\ Institut Camille Jordan, Université Claude Bernard Lyon 1, 43 boulevard du 11 novembre 1918 \\ F-69622 Villeurbanne Cedex, France \\ CRISTIANO RICCI \\ Email: cristiano.ricci@sns.it. \\ Scuola Normale Superiore, Piazza dei Cavalieri, 7, 56126 Pisa, Italy.
}

\begin{abstract}
An interacting particle system made of diffusion processes with local interaction is considered and the macroscopic limit to a nonlinear PDE is investigated. Few rigorous results exists on this problem and in particular the explicit form of the nonlinearity is not known. The paper reviews this subject, some of the main ideas to get the limit nonlinear PDE and provides both heuristic and numerical informations on the precise form of the nonlinearity which are new with respect to the literature and coherent with the few known informations.
\end{abstract}

Keywords: Evolution Equation; Phase Transition; Statistical Mechanic; Brownian Particle

AMS Subject Classification: 35Q70, 82C22, 60K35

\section{Introduction}

We are concerned with an interacting particle system governed by diffusions processes as follows:

$$
d X_{t}^{i, N}=-\sum_{j \neq i} \nabla V\left(X_{t}^{i, N}-X_{t}^{j, N}\right) d t+\sigma d B_{t}^{i}
$$

where $i=1, \ldots, K_{N}, X_{t}^{i, N} \in \mathbb{R}^{d}, V: \mathbb{R}^{d} \rightarrow \mathbb{R}$ has the form

$$
V(x)=U(|x|)
$$

where $U:(0, \infty) \rightarrow \mathbb{R}$ is twice differentiable, either with compact support or a suitable decay at infinity, $B_{t}^{i}$ are independent Brownian motions in $\mathbb{R}^{d}$ and $\sigma>0$.

The number $N$ is the scaling parameter and the number of particles $K_{N}$ will be assumed of order $N^{d}$. For mathematical simplicity we assume the particles live in a 
large torus

$$
\mathbb{T}_{N}^{d}:=\mathbb{R}^{d} / N \mathbb{Z}^{d}
$$

(the set $[0, N]^{d}$ with periodic identifications). When we assume

$$
K_{N}=\left\lfloor\rho N^{d}\right\rfloor
$$

for some $\rho>0$, we are saying that the density of particles in $\mathbb{T}_{N}^{d}$ is $\rho$.

We assume that the initial conditions $X_{0}^{i, N}, i=1, \ldots, N^{d}$, are random, independent, with a distributions such that the typical distance between neighbor particles is of order one, or $\frac{1}{\rho}$, but not concentrated with infinitesimal-in- $N$ interparticle distance. Expecting the same holds for $t>0$, each particle $X_{t}^{i, N}$ typically interacts only with a finite number of other particles in the case when $K$ is compact support (by finite number we mean finite in the limit when $N$ goes to infinity). Or, when $U$ has infinite support but decays suitably at infinity, although the number of particles seen by $X_{t}^{i, N}$ is infinite, only a finite number has a relevant influence on $X_{t}^{i, N}$. This is not a mean field regime; we call it local interaction regime. We shall also comment on intermediate situations between the two.

We want to investigate the macroscopic limit of this system, namely the weak limit of the empirical measure

$$
\mu_{t}^{N}=\frac{1}{N^{d}} \sum_{i=1}^{K_{N}} \delta_{\frac{1}{N} X_{t \cdot N^{2}}^{i, N}}
$$

corresponding to a parabolic zoom in space and time, natural because the transformation $B_{t}^{i} \rightarrow \frac{1}{N} B_{t \cdot N^{2}}^{i}$ leaves the law of Brownian motion invariant. Notice that $\mu_{t}^{N}$ is not a probability measure, unless $K_{N}=N^{d}$.

In the local interaction case considered here this limit is still poorly understood. In the works [14, 13], similarly to what happens for hydrodynamic limits of discrete systems [6], it is proved that the weak limit of the empirical measure $\mu_{t}^{N}$ is a weak solution of the following nonlinear Partial Differential Equation (PDE):

$$
\partial_{t} \rho=\frac{1}{2} \Delta P_{V}(\rho)
$$

but, apart from a number of restrictions on $\mathrm{V}$ imposed in these works, the main gap with respect to the discrete case is the lack of quantitative information on $P_{V}(\rho)$. In this exploratory work we review some facts known in the literature and present conjectures based on heuristic arguments and numerical simulations. We distinguish between the case when the interaction is purely repulsive, namely $U^{\prime}(r)<0$ for $r>0$ (possibly only up to some $r_{1}>0$ beyond which $U^{\prime}(r)=0$ ) and the case when the interaction includes an attractive component, namely $U^{\prime}(r)<0$ for $0<r<r_{0}$ and $U^{\prime}(r)>0$ for $r>r_{0}$ (again possibly only up to some $r_{1}>r_{0}$ beyond which $\left.U^{\prime}(r)=0\right)$. The repulsive case is better understood; the case with also local attraction is very difficult, with several obscure aspects.

Our motivation for studying this problem has been the desire to model adhesion between cells. A possible way of modeling such phenomenon is given by hard-core 
interacting particles. In this way particles are thought to be hard spheres which cannot compenetrate at all. Between the first results on the continuum limit for this type of particle system, we mention for one dimension [10 and for higher dimension 2]. A very recent result in this direction is [5]. The kind of interaction in which we are interested is the different from the one above mentioned. Assume a family of living cells is modeled simply by a position $X_{t}^{i, N}$ and a local interaction. Repulsion is motivated by a volume constraint: a cell is not a point, it has a finite size, and called $r_{0}$ its diameter, repulsion acts when the centers of the cells are at a distance smaller than $r_{0}$. But when they are at a distance slightly larger than $r_{0}$, cells do not simply separate: they are linked by macromolecules that produce adhesion between the cell membranes. We may steer the distance between the centers up so some value without splitting the cells, which are then subject to an attractive force. After some distance, the cells separate and do not feel each other anymore, corresponding to a compact support function $U$. Most of the literature describes cell adhesion by non-local attracting forces which are not realistic; they are a simplification (since they lead to mean field theories) and may give relatively good quantitative results when the mean field kernel has very short range, see [1], [3] [4]; but conceptually these models are wrong, since each cell interacts with infinitely many others and in a weak uniform way. The literature in biomathematics on cell adhesion seems to ignore the possibility, offered by the works of Varadhan [14 and Uchiyama [13, of studying the macroscopic limit of system like 1.1 having true local interaction. Our motivation for writing this work is to popularize this bibliographical link and propose additional quantitative conjectures beyond those made in [14, 13.

\section{Macroscopic view}

We now zoom and observe the previous particles as they were very close points in the unitary torus $\mathbb{T}^{d}=\mathbb{R}^{d} / \mathbb{Z}^{d}$ and we accelerate time according to the invariance of Brownian motion; we introduce the notations:

$$
\begin{aligned}
Y_{t}^{i, N} & :=\frac{1}{N} X_{t \cdot N^{2}}^{i, N} \in \mathbb{T}^{d} \\
W_{t}^{i} & :=\frac{1}{N} B_{t \cdot N^{2}}^{i}
\end{aligned}
$$

recalling that $W_{t}^{i}$ are independent Brownian motions. We have

$$
d Y_{t}^{i, N}=-N \sum_{j \neq i} \nabla V\left(X_{t \cdot N^{2}}^{i, N}-X_{t \cdot N^{2}}^{j, N}\right) d t+\sigma d W_{t}^{i}
$$

Set

$$
V_{N}(x):=N^{d} V(N x)=N^{d} U(|N x|)
$$

Then

$$
\frac{1}{N^{d}} \nabla V_{N}(x)=N \nabla V(N x)
$$


and thus we may write the previous rescaled equation in the elegant form

$$
d Y_{t}^{i, N}=-\frac{1}{N^{d}} \sum_{j \neq i} \nabla V_{N}\left(Y_{t}^{i, N}-Y_{t}^{j, N}\right) d t+\sigma d W_{t}^{i}
$$

which sounds like a mean field equation, due to the factor $\frac{1}{N^{d}}$, but it is not because the potential is rescaled (and it has infinitesimal range of interaction, in $\mathbb{T}^{d}$ ).

By Itô formula, if $\phi: \mathbb{T}^{d} \rightarrow \mathbb{R}$ is a smooth compact support test function, then

$$
\begin{aligned}
d \phi\left(Y_{t}^{i, N}\right) & =-(\nabla \phi)\left(Y_{t}^{i, N}\right) \frac{1}{N^{d}} \sum_{j \neq i} \nabla V_{N}\left(Y_{t}^{i, N}-Y_{t}^{j, N}\right) d t \\
& +(\nabla \phi)\left(Y_{t}^{i, N}\right) \sigma d W_{t}^{i}+\frac{\sigma^{2}}{2} \Delta \phi\left(Y_{t}^{i, N}\right) d t .
\end{aligned}
$$

Let us use a notational trick: the function $U$ is not defined for $r=0$, hence $V_{N}(0)$ is not defined; we set it equal to zerd ${ }^{\natural}$. Hence we may remove the restriction $j \neq i$ in the sum and write

$$
\begin{aligned}
d \phi\left(Y_{t}^{i, N}\right) & =-(\nabla \phi)\left(Y_{t}^{i, N}\right) \frac{1}{N^{d}} \sum_{j=1}^{K_{N}} \nabla V_{N}\left(Y_{t}^{i, N}-Y_{t}^{j, N}\right) d t+d R_{t}^{N, \phi} \\
& =-(\nabla \phi)\left(Y_{t}^{i, N}\right) \int_{\mathbb{T}^{d}} \nabla V_{N}\left(Y_{t}^{i, N}-y\right) \mu_{t}^{N}(d y) d t+d R_{t}^{N, \phi}
\end{aligned}
$$

where $d R_{t}^{N, \phi}=(\nabla \phi)\left(Y_{t}^{i, N}\right) \sigma d W_{t}^{i}+\frac{\sigma^{2}}{2} \Delta \phi\left(Y_{t}^{i, N}\right) d t$. Therefore

$$
d\left\langle\phi, \mu_{t}^{N}\right\rangle=-\left\langle\nabla \phi \int_{\mathbb{T}^{d}} \nabla V_{N}(\cdot-y) \mu_{t}^{N}(d y), \mu_{t}^{N}\right\rangle d t+\frac{\sigma^{2}}{2}\left\langle\Delta \phi, \mu_{t}^{N}\right\rangle d t+d M_{t}^{N, \phi}
$$

where the martingale $M_{t}^{N, \phi}$ is given by

$$
M_{t}^{N, \phi}=\int_{0}^{t} \frac{1}{N^{d}} \sum_{j=1}^{K_{N}}(\nabla \phi)\left(Y_{s}^{i, N}\right) \sigma d W_{s}^{(i)} .
$$

A classical simple computation with the isometry formula of Itô calculus proves that $M_{t}^{N, \phi}$ converges to zero in mean square; and also uniformly in time, using Doob's inequality. Assuming one can prove that $\mu_{t}^{N}$ converges weakly to a measure $\mu_{t}$, uniformly in time (maybe up to subsequences) and that $\mu_{t}$ has density $\rho_{t}$ with respect to Lebesgue measure (this is not necessary immediately, but will play a role later on), under the assumption that the same holds for the initial condition, we may pass to the limit in the terms $\left\langle\phi, \mu_{t}^{N}\right\rangle,\left\langle\phi, \mu_{0}^{N}\right\rangle, \int_{0}^{t}\left\langle\Delta \phi, \mu_{s}^{N}\right\rangle d s, M_{t}^{N, \phi}$; hence also the remaining term has a limit and we get

$$
\left\langle\phi, \rho_{t}\right\rangle=\left\langle\phi, \rho_{0}\right\rangle-\lim _{N \rightarrow \infty} \int_{0}^{t}\left\langle\nabla \phi \int_{\mathbb{T}^{d}} \nabla V_{N}(\cdot-y) \mu_{s}^{N}(d y), \mu_{s}^{N}\right\rangle d s+\frac{\sigma^{2}}{2} \int_{0}^{t}\left\langle\Delta \phi, \rho_{s}\right\rangle d s .
$$

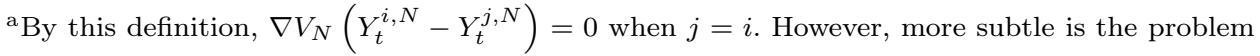
that we could have $Y_{t}^{i, N}-Y_{t}^{j, N}=0$ also sometimes for $j \neq i$. Fortunately, since we always assume to have a repulsive component in the interaction, one can prove this never happens . 
The main problem is to identify the limit left implicit above. For the purpose of the overview, we first identify the limit in the classical mean field case, Section 2.1 then we identify it in the case of repulsive integrable potential by means of simplified arguments not properly of local type, Sections 2.2, 2.3, and finally we discuss it in the main case motivating this paper, namely the case of local interaction, Section 3.

The tightness of the family of laws of $\mu^{N}$ required to implement rigorously the previous arguments is not trivial and it is discussed in [14, [13, under different conditions.

\subsection{Mean field interaction}

In broad terms, the mean field case is when we start directly in the unitary torus $\mathbb{T}^{d}$ with equations 2.1 but with $V_{N}$ independent of $N$ :

$$
V_{N}(x)=V(x) .
$$

Going back to formulation (1.1) in the large box $\mathbb{T}_{N}^{d}$, the potential there should depend on $N$. Thus the mean field case is not a particular case of the problem studied in this paper, since we started from (1.1) with a given potential. The only particular case satisfying $1.2,2.3$ and $V_{N}(x):=N^{d} V(N x)$ is the case

$$
V(x)=\frac{1}{\|x\|^{d}} .
$$

As a curiosity, this is the boundary case between weak and strong repulsion described in Section 3.1.

When (2.3) is imposed in equation 2.1), and $\nabla V$ is continuous and bounded, then tightness of the family of laws of $\mu^{n}$ is much easier [Sznitman] and convergence of the nonlinear term in 2.2 is almost trivial:

$$
\int_{\mathbb{T}^{d}} \nabla V_{N}(\cdot-y) \mu_{s}^{N}(d y) \rightarrow\left\langle\nabla V(\cdot-y), \rho_{s}\right\rangle=:\left(\nabla V * \rho_{s}\right)(y)
$$

and

$$
\int_{0}^{t}\left\langle\nabla \phi \int_{\mathbb{T}^{d}} \nabla V_{N}(\cdot-y) \mu_{s}^{N}(d y), \mu_{s}^{N}\right\rangle d s \rightarrow \int_{0}^{t}\left\langle\nabla \phi \cdot\left(\nabla V * \rho_{s}\right), \rho_{s}\right\rangle d s .
$$

Integrating (formally) by parts we get the mean field equation

$$
\partial_{t} \rho=\frac{\sigma^{2}}{2} \Delta \rho+\operatorname{div}(\rho(\nabla V * \rho)) .
$$

In applications, taking $V$ with very small support is a practical way to get numerical simulations very close to adhesion. But obviously the model required long range interaction, so it is logically incorrect, although reasonable under the rough view of a numerical simulation. 


\subsection{Two-step limit under integrable repulsive potential}

Although not logically correct, there is a cheap way to obtain a guess about the limit in 2.2. It is based on a limit taken in two successive steps. We introduce two scaling parameters $N$ and $M$ and replace the limit in 2.2 by

$$
\lim _{M \rightarrow \infty} \lim _{N \rightarrow \infty} \int_{0}^{t}\left\langle\nabla \phi \int_{\mathbb{T}^{d}} \nabla V_{M}(\cdot-y) \mu_{s}^{N}(d y), \mu_{s}^{N}\right\rangle d s .
$$

The first limit, in $N$, is like the mean field case (under the assumption that $\nabla V_{M}$ is continuous and bounded) and thus we get

$$
\lim _{M \rightarrow \infty} \int_{0}^{t}\left\langle\nabla \phi \cdot\left(\nabla V_{M} * \rho_{s}^{M}\right), \rho_{s}^{M}\right\rangle d s .
$$

Now assume a particular but natural version of the repulsive case: assume that $V$ is a probability density, of the form $V(x)=U(|x|)$ with $U$ decreasing on $(0, \infty)$. More precisely, assume it is such after normalization by

$$
C_{V}:=\int V(x) d x
$$

Then $C_{V}^{-1} V_{M}(x):=M^{d} C_{V}^{-1} V(M x)$ are classical mollifiers, with the property that

$$
\int_{\mathbb{T}^{d}} C_{V}^{-1} V_{M}(x-y) f(x) d x \rightarrow f(y)
$$

where convergence is for instance uniform on bounded sets when $f$ is uniformly continuous (several other results are known under different assumptions on $f$ ). Hence, assuming we can prove that $\rho_{s}^{M}$ converges to a limit $\rho_{s}$ in a suitable topology compatible with results of convergence of mollifiers (maybe up to subsequences), we have

$$
\begin{aligned}
\left(\nabla V_{M} * \rho_{s}^{M}\right)(y) & =\int_{\mathbb{T}^{d}} \nabla V_{M}(x-y) \rho_{s}^{M}(x) d x=-\int_{\mathbb{T}^{d}} V_{M}(x-y) \nabla \rho_{s}^{M}(x) d x \\
& \rightarrow-C_{V} \nabla \rho_{s}(y) \quad \text { as } M \rightarrow \infty
\end{aligned}
$$

and thus (up to rigorous care)

$$
\begin{aligned}
\lim _{M \rightarrow \infty} \int_{0}^{t}\left\langle\nabla \phi \cdot\left(\nabla V_{M} * \rho_{s}^{M}\right), \rho_{s}^{M}\right\rangle d s & =-C_{V} \int_{0}^{t}\left\langle\nabla \phi \cdot \nabla \rho_{s}, \rho_{s}\right\rangle d s \\
& =-\frac{C_{V}}{2} \int_{0}^{t}\left\langle\nabla \phi, \nabla \rho_{s}^{2}\right\rangle d s \\
& =\frac{C_{V}}{2} \int_{0}^{t}\left\langle\Delta \phi, \rho_{s}^{2}\right\rangle d s .
\end{aligned}
$$

Here we see for the first time the role of the density $\rho_{s}$ with respect to the measure $\mu_{s}(x)=\rho_{s}(x) d x$ : we need to take the square $\rho_{s}^{2}$, meaningful only for densities. The final equation, formally written after integration by parts, is

$$
\partial_{t} \rho=\frac{\sigma^{2}}{2} \Delta \rho+\frac{C_{V}}{2} \Delta \rho^{2}
$$


Notice that this result required $V$ integrable, $C_{V}<\infty$. Under this assumption, a result of Lemma 8.5 of 13 plus (8.10) of the same paper prove rigorously that the large $\rho$ asymptotic of the nonlinearity in 2.5 is precisely $\rho^{2}$. Our numerical simulations for repulsive integrable potentials confirm 2.5 also from other quantitative sides (not only the degree two for large $\rho$ ). Equation (2.5), in all its quantitative aspects, is also rigorously proved in the intermediate regime described in the next section. Therefore it seems that the simple conjecture based on the two-step method is quite realistic.

In order to validate this conjecture we present here some numerical results. Here we only briefly present the results, since the numerical analysis behind is more carefully explained in Section 4. We consider the following two potentials, whose plot is represented in Figure 1 (right):

$$
V(x)=\exp \left(-\frac{|x|^{2}}{2 \sigma^{2}}\right)
$$

and

$$
V(x)=\exp \left(-\frac{|x|^{2}}{2 \sigma^{2}}\right)\left(1-x^{3}\right) .
$$

In Figure 1 (left) we superimpose the function $P_{V}(\rho)=\sigma^{2} \rho+C_{V} \rho^{2}$, for $V$ equal to 2.7), with the function $P_{V}(\rho)$ computed numerically. As we can see there is almost perfect superimposition of the two, confirming our conjecture also for attractive potentials with negative sign, like 2.7). We also remark that simulations confirm that the leading term in the function $P_{V}(\rho)$ is of order $\rho^{2}$. It is however important to notice that Figure 1 contains information only for $\rho>1$. For smaller values of $\rho$ in fact there are some discrepancy between numerics and theory that are still not fully understood. For potential (2.6) we mention that the function $P_{V}(\rho)=\sigma^{2} \rho+C_{V} \rho^{2}$ and that computed numerically coincide also for values of $\rho$ smaller than one, confirming fully equation 2.5 as limiting equation. We also remark that a similar result holds, with the same constant $C_{V}$, in Bose-Einstein condensation theory, see 11.

\subsection{Intermediate interactions}

Karl Oelschläger, in a series of papers [7, 8, ,9, clarified rigorously the results when the interaction is intermediate between purely local and mean field. It is assumed that

$$
V_{N}(x):=N^{\beta d} V(N x)
$$

for some

$$
0<\beta<1
$$



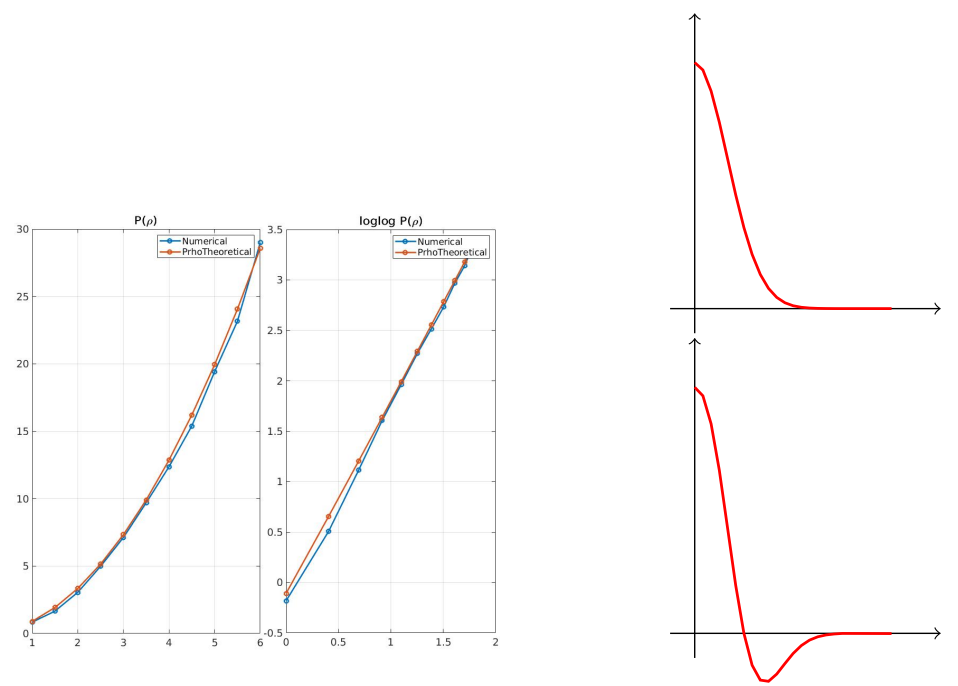

Figure 1: Left: Function $P_{V}(\rho)$ in the case of potential (2.7). Comparison between the function $P_{V}(\rho)$ obtained by numerical simulations and the function $\sigma^{2} \rho+C_{V} \rho^{2}$. Left: comparison between functions $P_{V}(\rho)$ in natural scale. Center: Comparison in $\log \log$ scale. Right: plot of the potentials in dimension one $V(x)$ equal to (2.6) (top) and (2.7) (bottom).

The case $\beta=1$ corresponds to local interaction, $\beta=0$ to mean field interaction. Intuitively, each particle $X_{t}^{i, N}$ interacts with infinitely many others but still with an infinitely small proportion of the total.

The potential $V$, in those works, is repulsive and integrable, as in the two-step approach described above. The final result is always equation (2.5). This is the best available confirmation that 2.5) is the correct one in the repulsive integrable regime and, as already said, our simulations confirm the result also in the limit case of local interactions.

\section{Local Interaction}

When the interaction potential $V$ is not integrable or not just repulsive, equation 2.5 seems to be false; it seems it should be replaced by an equation of the form

$$
\partial_{t} \rho=\frac{1}{2} \Delta P_{V}(\rho)
$$

where the nonlinear function $P_{V}(\rho)$ is close to linear for small values of $\rho$ (like $\left.\sigma^{2} \Delta \rho+C_{V} \Delta \rho^{2}\right)$ but growth more than quadratically as $\rho \rightarrow \infty$ and, when there is also suitable attraction, the slope for small $\rho$ may be different from $\sigma^{2}$. The first rigorous proof that $(3.1)$ is the macroscopic limit of system (1.1) has been given in $d=1$ by Varadhan 14 in the case of repulsive potential, without a quantification of $P_{V}(\rho)$. In general dimension, the basic result has been proved by Uchiyama 13; it is a conditional result (conditional because it is based on an ergodic assumption which is an open problem) but holds for quite general potentials, which may include also an attractive part; and in the Appendix, in particular Lemma 8.5 (see also (8.10)), 13 provides quantitative result of the form $P_{V}(\rho)=O\left(\rho^{\gamma}\right)$ as $\rho \rightarrow \infty$ with 
precise prescription of $\gamma$ depending on the singularity of $V$ at $x=0$. Thus, although being only a one-side result (because it is of the form $O\left(\rho^{\gamma}\right)$ ) and it is only for large $\rho$, it is a precious indications. Not only it is the only quantitative rigorous result but also the order $\gamma$ of the power is confirmed by our numerical simulations to be the true one, not only a bound on one side.

Below, our goal is to give more explicit forms of $P_{V}(\rho)$, coherent with [13], obtained when possible by both heuristic arguments and numerical simulations.

\subsection{Some remark on the potential}

As already remarked above, we have in mind sometimes the purely repulsive case, since it is easier, and sometime else the attractive-repulsive case, motivated for instance by cell adhesion. Let us describe the attractive-repulsive case. The potential $U$ is made of two components, a repulsive one corresponding to volume constraint $V_{v o l}$ and an attractive one corresponding to adhesion $V_{a d h}$

$$
U(r)=U_{v o l}(r)+U_{a d h}(r) .
$$

where $U, U_{a d h}, U_{v o l}:(0,+\infty) \rightarrow \mathbb{R}$ are piecewise $C^{1}$ functions with the properties

$$
U_{v o l}^{\prime} \begin{cases}<0 & \text { if } r \in\left(0, r_{1}\right) \\ =0 & \text { if } r>r_{1}\end{cases}
$$

and

$$
U_{a d h}^{\prime} \begin{cases}<0 & \text { if } r \in\left(0, r_{0}\right) \\ >0 & \text { if } r \in\left(r_{0}, r_{1}\right) \\ =0 & \text { if } r>R_{1} .\end{cases}
$$

This is the general form in the compact support case, but we shall also consider the case when both $U_{a d h}$ and $U_{v o l}$ have full support but suitable decay at infinity, like the case of Lennard-Jones potential.

As mentioned several times above, the most relevant results on macroscopic limit for local interaction are given by 14$]$ and $[13$. In both paper the result of convergence is stated under some condition on the potential, see for instance page 1160 of [13], which include both the case of a purely repulsive potential $U(r)=U_{v o l}(r)$ and the case of attractive-repulsive one $U(r)=U_{v o l}(r)+U_{a d h}(r)$. For certain results a main distinction is made by the following condition: we call weak repulsion the case when $\int_{0}^{1} U(r) r^{d-1} d r<\infty$, strong repulsion the case when $\int_{0}^{1} U(r) r^{d-1} d r=\infty$.

\subsection{Manipulation of the nonlinear term}

Apart from the tightness problem that we address to the literature, the main problem left open above is to understand the limit of nonlinear term in equation 2.2

$$
\left\langle\mu_{t}^{N}, \nabla \phi \cdot \nabla\left(V_{N} * \mu_{t}^{N}\right)\right\rangle=\left\langle\nabla \phi \int_{\mathbb{T}^{d}} \nabla V_{N}(\cdot-y) \mu_{t}^{N}(d y), \mu_{t}^{N}\right\rangle .
$$


Let us assume compact support potential, being $r_{1}$ the size of the support as in the examples above. Let us rewrite the nonlinear term explicitly as (assuming also $\left.K_{N}=\left[N^{d} \rho\right]\right)$

$$
=\frac{1}{N^{d}} \sum_{i=1}^{\left[N^{d} \rho\right]} \nabla \phi\left(Y_{t}^{i, N}\right) \cdot N \sum_{j:\left|Y_{t}^{i, N}-Y_{t}^{j, N}\right| \leq \frac{r_{1}}{N}} U^{\prime}\left(N\left|Y_{t}^{i, N}-Y_{t}^{j, N}\right|\right) \frac{Y_{t}^{i, N}-Y_{t}^{j, N}}{\left|Y_{t}^{i, N}-Y_{t}^{j, N}\right|}
$$

or also as

$$
=\frac{1}{N^{2 d}} \sum_{i, j=1}^{\left[N^{d} \rho\right]} \nabla \phi\left(Y_{t}^{i, N}\right) \cdot \nabla V_{N}\left(Y_{t}^{i, N}-Y_{t}^{j, N}\right) .
$$

We shall use both expressions.

There is a cancellation we need to implement; systems with this cancellation are called gradient systems in other contexts [6]; and the same cancellation is used in Schoquet symmetrization approach to measure valued solutions to 2D Euler equations 12. Under our assumptions we have

$$
\nabla V_{N}(-x)=-\nabla V_{N}(x)
$$

Observe two particles $X_{t}^{i}$ and $X_{t}^{j}$ : the "force" impressed by $X_{t}^{j}$ on $X_{t}^{i}$ is

$$
-\frac{1}{N^{d}} \nabla V_{N}\left(Y_{t}^{i, N}-Y_{t}^{j, N}\right) \quad\left(\text { force of } X_{t}^{j} \text { on } X_{t}^{i}\right. \text { ) }
$$

while the force impressed by $X_{t}^{i}$ on $X_{t}^{j}$ is

$$
-\frac{1}{N^{d}} \nabla V_{N}\left(Y_{t}^{i, N}-Y_{t}^{j, N}\right)=\frac{1}{N^{d}} \nabla V_{N}\left(Y_{t}^{i, N}-Y_{t}^{j, N}\right) \quad\left(\text { force of } Y_{t}^{j, N} \text { on } Y_{t}^{i, N}\right) .
$$

In the equations of motion these two forces never appear together, but in the formula for $\left\langle S_{t}^{N}, \nabla \phi \cdot \nabla\left(V_{N} * S_{t}^{N}\right)\right\rangle$ the analogous terms appear together, precisely as:

$$
\begin{aligned}
& \nabla \phi\left(Y_{t}^{i, N}\right) \cdot \nabla V_{N}\left(Y_{t}^{i, N}-Y_{t}^{j, N}\right)+\nabla \phi\left(Y_{t}^{j, N}\right) \cdot \nabla V_{N}\left(Y_{t}^{j, N}-Y_{t}^{i, N}\right) \\
& =\left(\nabla \phi\left(Y_{t}^{i, N}\right)-\nabla \phi\left(Y_{t}^{j, N}\right)\right) \cdot \nabla V_{N}\left(Y_{t}^{i, N}-Y_{t}^{j, N}\right) .
\end{aligned}
$$

Since $\phi$ is smooth, and only nearby particles interact, this is almost a cancellation. By Taylor formula it is approximatively equal to

$$
\begin{aligned}
& =\sum_{\alpha=1}^{d} \partial_{\alpha}\left(\phi\left(Y_{t}^{i, N}\right)-\phi\left(Y_{t}^{j, N}\right)\right) \partial_{\alpha} V_{N}\left(Y_{t}^{i, N}-Y_{t}^{j, N}\right) \\
& \sim \sum_{\alpha, \beta=1}^{d} \partial_{\alpha} \partial_{\beta} \phi\left(Y_{t}^{j, N}\right) \partial_{\alpha} V_{N}\left(Y_{t}^{i, N}-Y_{t}^{j, N}\right)\left(Y_{t}^{i, N}-Y_{t}^{j, N}\right)_{\beta} .
\end{aligned}
$$

The approximation is reasonable because $\left|Y_{t}^{i, N}-Y_{t}^{j, N}\right| \leq \frac{r_{1}}{N}$. 
Let us put this approximation in the full nonlinear expression:

$$
\begin{aligned}
\left\langle S_{t}^{N}, \nabla \phi \cdot \nabla\left(V_{N} * S_{t}^{N}\right)\right\rangle & =\frac{1}{N^{2 d}} \sum_{i<j} \sum_{\alpha, \beta=1}^{d} \partial_{\alpha} \partial_{\beta} \phi\left(Y_{t}^{j, N}\right) \partial_{\alpha} V_{N}\left(Y_{t}^{i, N}-Y_{t}^{j, N}\right)\left(Y_{t}^{i, N}-Y_{t}^{j, N}\right)_{\beta} \\
& =\frac{1}{2 N^{2 d}} \sum_{i, j=1}^{\left[N^{d} \rho\right]} \sum_{\alpha, \beta=1}^{d} \partial_{\alpha} \partial_{\beta} \phi\left(Y_{t}^{j, N}\right) \partial_{\alpha} V_{N}\left(Y_{t}^{i, N}-Y_{t}^{j, N}\right)\left(Y_{t}^{i, N}-Y_{t}^{j, N}\right)_{\beta} .
\end{aligned}
$$

Let us introduce the function

$$
\psi_{\alpha \beta}(x)=x_{\beta} \partial_{\alpha} V(x) .
$$

Recall $V_{N}(x)=N^{d} V(N x)$, hence $\nabla V_{N}(x)=N N^{d}(\nabla V)(N x)$, hence

$$
\begin{aligned}
\partial_{\alpha} V_{N}\left(Y_{t}^{i, N}-Y_{t}^{j, N}\right)\left(Y_{t}^{i, N}-Y_{t}^{j, N}\right)_{\beta} & =N N^{d}\left(\partial_{\alpha} V\right)\left(N\left(Y_{t}^{i, N}-Y_{t}^{j, N}\right)\right)\left(Y_{t}^{i, N}-Y_{t}^{j, N}\right)_{\beta} \\
& =N^{d} \psi_{\alpha \beta}\left(N\left(Y_{t}^{i, N}-Y_{t}^{j, N}\right)\right)
\end{aligned}
$$

therefore

$$
\begin{aligned}
& \left\langle\mu_{t}^{N}, \nabla \phi \cdot \nabla\left(V_{N} * \mu_{t}^{N}\right)\right\rangle=\frac{1}{2 N^{2 d}} \sum_{i, j=1}^{\left[N^{d} \rho\right]} \sum_{\alpha, \beta=1}^{d} \partial_{\alpha} \partial_{\beta} \phi\left(Y_{t}^{j, N}\right) N^{d} \psi_{\alpha \beta}\left(N\left(Y_{t}^{i, N}-Y_{t}^{j, N}\right)\right) \\
& =\frac{1}{2 N^{d}} \sum_{i=1}^{\left[N^{d} \rho\right]} \sum_{\alpha, \beta=1}^{d} \partial_{\alpha} \partial_{\beta} \phi\left(Y_{t}^{j, N}\right) \sum_{j:\left|Y_{t}^{i, N}-Y_{t}^{j, N}\right| \leq \frac{r_{1}}{N}} \psi_{\alpha \beta}\left(N\left(Y_{t}^{i, N}-Y_{t}^{j, N}\right)\right) .
\end{aligned}
$$

It remains to understand where this expression converges.

\subsection{Invariant measures}

Let us discuss invariant measures for the original microscopic system 1.1) on the large torus $\mathbb{T}_{N}^{d}$. Let us parametrize this system in a more general way. Given $\rho>0$ and $L>0$, on the torus $\mathbb{T}_{L}^{d}=\mathbb{R}^{d} / L \mathbb{Z}^{d}$ (informally $[0, L]^{d}$ ) consider $K_{L}:=\left\lfloor L^{d} \rho\right\rfloor$ particles $X_{t}^{i}$. The SDE system has the invariant measure

$$
\mu_{\rho, L}\left(d x^{1} \ldots d x^{K_{L}}\right)=\frac{1}{Z_{\rho, L}} \exp \left(-\frac{1}{2} \sum_{i, j=1}^{K_{L}} V\left(x^{i}-x^{j}\right)\right) d x^{1} \ldots d x^{K_{L}} .
$$

Taken $\psi \in C_{c}^{0}\left(\mathbb{R}^{d}\right)$ (it will be one of the functions $\psi_{\alpha \beta}(x)=x_{\beta} \partial_{\alpha} V(x)$ introduced above), assume we are able to prove that there exists $\Psi_{V}: \mathbb{R} \rightarrow \mathbb{R}$ such that

$$
\lim _{L \rightarrow \infty} \int_{\left(\mathbb{T}_{L}^{d}\right)^{K_{L}}}\left|\frac{1}{L^{d}} \sum_{i, j=1}^{K_{L}} \psi\left(x^{i}-x^{j}\right)-\Psi_{V}(\rho)\right|^{2} \mu_{L}\left(d x^{1} \ldots d x^{K_{L}}\right)=0 .
$$


If this happens, we could say that "spatial averages of local observables converge". This is a very technical result but heuristically $\frac{1}{L^{d}} \sum_{i, j=1}^{K_{L}} \psi\left(x^{i}-x^{j}\right)$ is an average:

$$
\begin{aligned}
\frac{1}{L^{d}} \sum_{i, j=1}^{K_{L}} \psi\left(x^{i}-x^{j}\right) & =\frac{1}{L^{d}} \sum_{i=1}^{K_{L}} F(x) \\
F(x) & :=\sum_{\left|x^{i}-x^{j}\right| \leq r_{1}} \psi\left(x^{i}-x^{j}\right)
\end{aligned}
$$

with the notation $x=\left(x^{1}, \ldots, x^{K_{L}}\right)$; property 3.2 is a sort of Law of Large Numbers (or more precisely a spatial ergodic property) with respect to the Gibbs measure in infinite volume.

Remark 3.1. In discrete systems like those considered in 6 , the invariant measures are much easier, usually product measures and the corresponding quantities can be computed more explicitly. The weak point of the continuum theory is the difficulty to compute $\Psi_{V}(\rho)$.

\subsection{Local equilibrium}

Definition 3.1. Let a continuous function $\rho:[0, T] \times \mathbb{T}^{d} \rightarrow \mathbb{R}$ with constant in time mass $\bar{\rho}=\int_{\mathbb{T}^{d}} \rho_{t}(y) d y$ be given. For particle system 2.1 with $K_{N}=\left[\bar{\rho} N^{d}\right]$, we say that local equilibrium holds if, for every $\varphi, \psi \in C\left(\mathbb{T}^{d}\right)$, every $t \in[0, T]$, in probability we have

$\lim _{N \rightarrow \infty} \frac{1}{N^{d}} \sum_{i=1}^{\left[\bar{\rho} N^{d}\right]} \varphi\left(Y_{t}^{j, N}\right) \sum_{j:\left|Y_{t}^{i, N}-Y_{t}^{j, N}\right| \leq \frac{r_{1}}{N}} \psi\left(N\left(Y_{t}^{i, N}-Y_{t}^{j, N}\right)\right)=\int_{\mathbb{T}^{d}} \varphi(y) \Psi_{V}\left(\rho_{t}(y)\right) d y$.

Assume this property is satisfied. Let us apply it to $\varphi=\partial_{\alpha} \partial_{\beta} \phi, \psi=\psi_{\alpha \beta}$. We get

$$
\begin{aligned}
& \lim _{N \rightarrow \infty} \frac{1}{2 N^{d}} \sum_{i=1}^{\left[\bar{\rho} N^{d}\right]} \partial_{\alpha} \partial_{\beta} \phi\left(Y_{t}^{j, N}\right) \sum_{j:\left|Y_{t}^{i, N}-Y_{t}^{j, N}\right| \leq \frac{r_{1}}{N}} \psi_{\alpha \beta}\left(N\left(Y_{t}^{i, N}-Y_{t}^{j, N}\right)\right) \\
& =\frac{1}{2} \int_{\mathbb{T}^{d}} \partial_{\alpha} \partial_{\beta} \phi(x) \Psi_{V}^{\alpha, \beta}\left(\rho_{t}(x)\right) d x
\end{aligned}
$$

where $\Psi_{V}^{\alpha, \beta}$ is defined from $\psi_{\alpha \beta}$ via 3.2 . hence

$$
\frac{d}{d t}\left\langle\rho_{t}, \phi\right\rangle+\frac{1}{2} \int_{\mathbb{T}^{d}} \sum_{\alpha, \beta} \partial_{\alpha} \partial_{\beta} \phi(x) \Psi_{V}^{\alpha, \beta}\left(\rho_{t}(x)\right) d x=\frac{\sigma^{2}}{2}\left\langle\rho_{t}, \Delta \phi\right\rangle .
$$

By an isotropy argument that we omit we finally get

$$
\frac{d}{d t}\left\langle\rho_{t}, \phi\right\rangle+\frac{1}{2} \int_{\mathbb{T}^{d}} \Delta \phi(x) \Psi_{V}\left(\rho_{t}(x)\right) d x=\frac{\sigma^{2}}{2}\left\langle\rho_{t}, \Delta \phi\right\rangle
$$


and by formal integration by parts

$$
\partial_{t} \rho_{t}=\frac{\sigma^{2}}{2} \Delta \rho_{t}-\frac{1}{2} \Delta \Psi_{V}\left(\rho_{t}\right)
$$

where $\Psi_{V}(\rho)$ is $\Psi_{V}^{\alpha, \alpha}(\rho)$ independently of $\alpha=1, \ldots, d$.

\subsection{On local equilibrium: a multiscale argument}

It remains to understand the meaning of 3.1 and how to prove its validity. This is well understood in the discrete setting but not so much in the continuous SDE one. Let us discuss the property

$$
\lim _{N \rightarrow \infty} \frac{1}{N^{d}} \sum_{i, j} \varphi\left(Y_{t}^{j, N}\right) \psi\left(N\left(Y_{t}^{i, N}-Y_{t}^{j, N}\right)\right)=\int_{\mathbb{T}^{d}} \varphi(x) \Psi_{V}\left(\rho_{t}(x)\right) d x .
$$

Since it will appear several times below, let us write

$$
A_{N, t}:=\frac{1}{N^{d}} \sum_{i, j} \varphi\left(Y_{t}^{j, N}\right) \psi\left(N\left(Y_{t}^{i, N}-Y_{t}^{j, N}\right)\right) .
$$

Decompose $\mathbb{T}^{d}=[0,1]^{d}$ in $m_{N}$ squares $Q_{N}\left(y_{1}^{N}\right), \ldots, Q_{N}\left(y_{m_{N}}^{N}\right)$ of the form

$$
Q_{N}\left(y_{k}^{N}\right)=\prod_{\alpha=1}^{d}\left[y_{k, \alpha}^{N}-\frac{1}{2 m_{N}}, y_{k, \alpha}^{N}+\frac{1}{2 m_{N}}\right] \quad k=1, \ldots, m_{N}
$$

where $y_{k}^{N}$ are points of coordinates $y_{k, \alpha}^{N}, \alpha=1, \ldots, d$. The idea is that each one contains a huge number of particles, but $m_{N}$ is very large as well. Hence assume

$$
\begin{aligned}
& m_{N} \rightarrow \infty \quad \text { as } N \rightarrow \infty \\
& m_{N}=o\left(N^{d}\right) .
\end{aligned}
$$

Let us suppose the interactions between particles lying in different domains have a small contribution. Thus let us approximate

$$
A_{N, t} \sim \frac{1}{m_{N}} \sum_{k=1}^{m_{N}} \frac{m_{N}}{N^{d}} \sum_{Y_{t}^{i, N}, Y_{t}^{j, N} \in Q_{N}\left(y_{k}^{N}\right)} \varphi\left(Y_{t}^{j, N}\right) \psi\left(N\left(Y_{t}^{i, N}-Y_{t}^{j, N}\right)\right) .
$$

Since the size $\frac{1}{m_{N}}$ of the little squares is very small, approximatively

$$
\varphi\left(Y_{t}^{j, N}\right) \sim \varphi\left(y_{k}^{N}\right) \quad \text { when } Y_{t}^{j, N} \in Q_{N}\left(y_{k}^{N}\right)
$$

and thus let us approximate further

$$
A_{N, t} \sim \frac{1}{m_{N}} \sum_{k=1}^{m_{N}} \varphi\left(y_{k}^{N}\right)\left[\frac{m_{N}}{N^{d}} \sum_{Y_{t}^{i, N}, Y_{t}^{j, N} \in Q_{N}\left(y_{k}^{N}\right)} \psi\left(N\left(Y_{t}^{i, N}-Y_{t}^{j, N}\right)\right)\right] .
$$


The external average $\frac{1}{m_{N}} \sum_{k=1}^{m_{N}}$ can be seen as the Riemann sums approximating an integral, yielding

$$
A_{N, t} \sim \int_{\mathbb{T}^{d}} \varphi(y)\left[\frac{m_{N}}{N^{d}} \sum_{Y_{t}^{i, N}, Y_{t}^{j, N} \in Q_{N}(y)} \psi\left(N\left(Y_{t}^{i, N}-Y_{t}^{j, N}\right)\right)\right] d y
$$

Thus we have to understand the expression

$$
\frac{m_{N}}{N^{d}} \sum_{Y_{t}^{i, N}, Y_{t}^{j, N} \in Q_{N}(y)} \psi\left(N\left(Y_{t}^{i, N}-Y_{t}^{j, N}\right)\right)
$$

when $N \rightarrow \infty$. Recalling the definition $Y_{t}^{i, N}:=\frac{1}{N} X_{N^{2} t}^{i, N}$, the last expression is equal to

$$
\underset{X_{N^{2} t}^{i, N}, X_{N^{2} t}^{j, N} \in \prod_{\alpha=1}^{d}\left[N y_{\alpha}-\frac{N}{2 m_{N}}, N y_{\alpha}+\frac{N}{2 m_{N}}\right]}{ } \psi\left(X_{N^{2} t}^{i, N}-X_{N^{2} t}^{j, N}\right) .
$$

Time is much accelerated and thus it is reasonable to approximate $\left\{X_{N^{2} t}^{i}\right\}$ with the corresponding stationary process; by the ergodic property $(3.2)$, the previous expression, computed along realizations of the stationary process, is close to $\Psi_{V}\left(\rho_{t}(x)\right)$. The choice of the density $\rho_{t}(x)$ is due to the fact that the number of particles in $Q_{N}(y)$ is approximately given by $\rho_{t}(x) \cdot N / m_{N}$.

\subsection{Summary of the main result}

We have heuristically shown above (proofs are given in [13, $[14]$ ) that the macroscopic limit of the particle system with local interaction is the nonlinear PDE

$$
\partial_{t} \rho_{t}=\frac{1}{2} \Delta P_{V}\left(\rho_{t}\right)
$$

where

$$
P_{V}(\rho):=\sigma^{2} \rho-\Psi_{V}(\rho)
$$

and $\Psi_{V}(\rho)$ is given by

$$
\Psi_{V}(\rho)=\lim _{L \rightarrow \infty} \frac{1}{L^{d}} \sum_{i, j=1}^{K_{L}} \psi_{\alpha \alpha}\left(x^{i}-x^{j}\right)
$$

for any $\alpha=1, \ldots, d$,

$$
\psi_{\alpha \alpha}(x)=x_{\alpha} \partial_{\alpha} V(x) .
$$

In (3.3) the sample $x^{1}, \ldots, x^{K_{L}}$ must be taken distributed according to $\mu_{\rho, L}$, see Section 3.3 and in particular see property $(3.2)$ which explains the meaning of the limit in (3.3). In numerical simulations we shall simulate system (1.1) in a large periodic box and take the tail of the simulation as a sample approximately distributed as $\mu_{\rho, L}$, heuristically relying on a result of ergodicity in time. 


\section{Quantitative results and conjectures about $\boldsymbol{P}_{V}(\rho)$}

Our aim is to get some information of the function $P_{V}(\rho)$. Our idea to estimate $P_{V}(\rho)$ is to approximate the rhs of 3.2 with one of the realization of $\left(y^{1}, \cdots, y^{N}\right)$ according to the invariant measure $\mu_{\rho, L}$ :

$$
P_{V}(\rho)--\sigma^{2} \rho \approx \lim _{L \rightarrow \infty} \frac{1}{L^{d}} \sum_{i, j=1}^{N_{\rho, L}} \psi\left(x^{i}-x^{j}\right) .
$$

with $\left(x^{1}, \cdots, x^{N}\right)$ realization of the variables $\left(y^{1}, \ldots, y^{N}\right)$, distributed according to $\mu_{\rho, L}$. At this point it is clear that the key to the study of the virial formula consists in the realization of variables $\left(y^{1}, \ldots, y^{N}\right)$. In the paper we propose two way of visualizing the Gibbs measure: some heuristic arguments followed (and confirmed) by numerical simulations. To produce samples of the Gibbs measure is not a simple task. However, assuming a suitable ergodicity property, we can produce realizations of such measure $\mu^{\rho, L}$ by simulations on large times of a SDE whose invariant measure is $\mu^{\rho, L}$. In a general framework, if $X_{t}$ denotes the solution of the SDE whose invariant measure is $\mu$, then the time average

$$
\frac{1}{T} \int_{t_{0}}^{t_{0}+T} \psi\left(X_{s}\right) d s
$$

by ergodicity property is a good approximation of the spatial integral

$$
\int \psi(x) \mu(d x) \text {. }
$$

Now, applying this idea to our case: the SDE whose invariant measure is $\mu^{\rho, L}$ is given by the system of SDEs

$$
d X^{i, N}=-\sum_{j=1, j \neq i}^{N^{\rho, L}} \nabla U\left(X_{t}^{j, N}-X_{t}^{i, N}\right) d t+\sigma d B_{t}^{i}
$$

in the torus $\mathbb{T}_{L}^{d}$. We perform simulations both in dimensions one and two, even if we focus more on the one dimensional case for a matter of simplicity.

Both for the heuristic and the numerics we follow the same strategy: we start by making an educated guess about the invariant measure $\mu_{\rho, L}$ depending on the potential $U$ and the density $\rho$. In particular we look for an equilibrium configuration $\left(\bar{x}^{i, N}\right)_{i=1, \ldots, N}$ for a system of deterministic ODEs satisfying

$$
\dot{x}_{t}^{i, N}=-\sum_{j=1, j \neq i}^{N^{\rho, L}} \nabla V\left(x_{t}^{j, N}-x_{t}^{i, N}\right)
$$

which the same as system 4.2 taking $\sigma=0$. This equilibrium configuration is computed directly for some particular choices of the potential. Of course in some situations multiple equilibrium configurations may exists. In those cases we have to rely on a more intuitive reasoning on what the stationary distribution could be. These deterministic equilibrium points are then used for two different purposes. For 
the numerics, they are used as initial condition for the random dynamics 4.2 , since we have the intuition that they represents already a good approximation of the invariant measure. Moreover we use the same points as fixed particle positions in the virial formula (4.1) to obtain an explicit expression for $P_{V}(\rho)$. By approximating

$$
\mu_{\rho, L} \approx \frac{1}{N} \sum_{i=1}^{N} \delta_{\bar{x}^{i, N}}
$$

we can carry out the computational analytically and compare the formula obtained with the values coming from numerical simulations.

In the next sections we presents the results obtained by the two approaches. In particular we analyze the following two main examples for the potential. We will call the first one the purely repulsive case. It is defined by

$$
V(x)=\left(\frac{1}{\alpha|x|^{\alpha}}-C\right) \mathbb{1}_{|x| \leq R_{1}},
$$

where the constant $C$ is chosen in order to make the function $V$ continuous in $|x|=R_{1}$ and for $\alpha>0$. It represents the ideal repulsive potential for two solid object that interact by contact forces. Of course what we have in mind is not the case of hard-core interaction between hard spheres, which when touch cannot compenetrate. The example we think of is more that of biological cells, that when touch can stretch an compress a bit one into the other. Moreover we also consider an attractive-repulsive case, defined by

$$
V(x)=\left(\frac{R_{0}^{\alpha}}{\alpha|x|^{\alpha}}-\frac{R_{0}^{\beta}}{\beta|x|^{\beta}}+C\right) \mathbb{1}_{|x| \leq R_{1}},
$$

where the constant $C$ is chosen in order to make the function $V$ continuous in $|x|=R_{1}, \alpha>\beta$ and $R_{1}>R_{0}$. This potential has the same shape of the classical Lennard-Jones potential but restricted to have finite range. In this case we target specifically the phenomenon of cell-cell adhesion, where cells repulse each other when too close (due to contact forces) and tends to stick one to each other when they touch. At longer than contact range there is no interaction, motivating the truncation in the potential $V$.

In both case we carry out all the analytical computations with more details in dimension one. In higher dimension we only analyze the asymptotic of $P_{V}(\rho)$ and perform numerical simulation to dimension up to two.

\subsection{Purely repulsive case}

Let us start by the purely repulsive case. We now discuss what are the possible equilibrium points of 4.3 for different values of $\rho$. Let assume that we work in dimension $d=1$ and for simplicity imagine first the case where $R_{1}=+\infty$. Now it is clear that particles have the tendency to stay as far as possible from each other. 


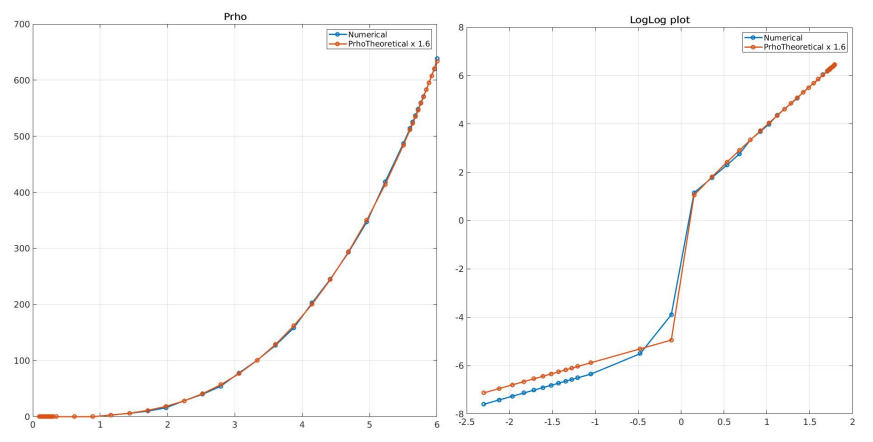

Figure 2: Purely repulsive case with compact support, $\alpha=2, R_{1}=1$ in dimension $d=1$. Comparison between the function $P_{V}(\rho)$ obtained by numerical simulations and by that obtained in Claim 1. Left: comparison between functions $P_{V}(\rho)$ in natural scale. Right: Comparison in log log scale. The theoretical function computed as in Claim 1 has been rescaled by 1.6 .

Assuming a density $\rho$ then the average distance, in a torus of size $L$ is of order $1 / \rho$. Hence we approximate

$$
\mu_{\rho, L} \approx \frac{1}{N} \sum_{i=1}^{N} \delta_{\bar{x}^{i, N}}
$$

with

$$
\bar{x}^{i, N}=\frac{i}{\rho} \quad i=1, \ldots, N
$$

see Figure 4. If $R_{1}<\infty$, hence the potential has compact support, in order for particle to interact with each other one needs to have $1 / \rho<R_{1}$, in other terms $\rho>1 / R_{1}$. We figure out that the behavior of particles is not so different from the long range case. Let us focus on the low density configuration $\rho<1 / R_{1}$ and let us assume particles to be at equal distance $1 / \rho$. We can think that each Brownian particle $X^{i}$ has attached to itself a sort of delimitation zone of amplitude $R_{1}$, $\left[X^{i}-R_{1} / 2, X^{i}+R_{1} / 2\right]$ and as soon another particle goes inside such area, it is pushed away. So at the end particles will always come back to the equal distance configuration. For $\rho>1 / R_{1}$ we expect particle to stay as large as possible. So also for the compactly supported case we assume that 4.6 holds.

Claim 1. In dimension $d=1$, if the potential $U$ is given by (4.4) we claim that

$$
P_{V}(\rho) \approx \begin{cases}\sigma^{2} \rho & \rho<1 / R_{1} \\ \sigma^{2} \rho+\frac{\alpha}{\alpha-1} \rho^{1+\alpha}-\frac{1}{R_{1}^{\alpha-1}(\alpha-1)} \rho^{2} & \rho \geq 1 / R_{1}\end{cases}
$$


for $\alpha \neq 1$. Instead for $\alpha=1$ we claim

$$
P_{V}(\rho) \approx \begin{cases}\sigma^{2} \rho & \rho<1 / R_{1}, \\ \sigma^{2} \rho+\rho^{2}\left(\log (\rho)+\log \left(R_{1}\right)+1\right) & \rho \geq 1 / R_{1} .\end{cases}
$$

Let us start by discussing the case $\rho<1 / R_{1}$. By using equation (4.1) with points $\bar{x}^{i, N}$ we obtain

$$
-\frac{1}{L} \sum_{i=1}^{N_{\rho, L}} \sum_{j=1}^{N_{\rho, L}} V^{\prime}\left(\left|\bar{x}^{i, N}-\bar{x}^{j, N}\right|\right)\left|\bar{x}^{i, N}-\bar{x}^{j, N}\right|=-\rho \sum_{i=1}^{\rho L} V^{\prime}\left(\left|\bar{x}^{i, N}\right|\right)\left|\bar{x}^{i, N}\right|
$$

since $N_{\rho L}=\rho L$ and particles are all at the same distance one from the other. Moreover we have

$$
-\rho \sum_{i=1}^{\rho L} V^{\prime}\left(\left|\bar{x}^{i, N}\right|\right)\left|\bar{x}^{i, N}\right|=\rho \sum_{i=1}^{\rho L} \frac{\rho^{\alpha+1}}{i^{\alpha+1}} \frac{i}{\rho} \mathbb{1}_{\left|\frac{i}{\rho}\right| \leq R_{1}}=0
$$

since we assumed $1 / \rho>R_{1}$. In the case $\rho>1 / R_{1}$ we can carry out the computation (assume $\alpha \neq 1$ for simplicity) obtaining

$$
\rho \sum_{i=1}^{\rho L} \frac{\rho^{\alpha+1}}{i^{\alpha+1}} \frac{i}{\rho} \mathbb{1}_{\left|\frac{i}{\rho}\right| \leq R_{1}}=\rho^{1+\alpha} \sum_{i=1}^{\rho R_{1}} \frac{1}{i^{\alpha}} .
$$

Now we approximate

$$
\sum_{i=1}^{\rho R_{1}} \frac{1}{i^{\alpha}} \approx 1+\int_{1}^{\rho R_{1}} \frac{1}{x^{\alpha}} d x=1+\frac{1}{\alpha-1}\left(1-\frac{1}{R_{1}^{\alpha-1} \rho^{\alpha-1}}\right),
$$

hence

$\rho^{1+\alpha} \sum_{i=1}^{\rho R_{1}} \frac{1}{i^{\alpha}}=\rho^{1+\alpha}\left[1+\frac{1}{\alpha-1}\left(1-\frac{1}{R_{1}^{\alpha-1} \rho^{\alpha-1}}\right)\right]=\frac{\alpha}{\alpha-1} \rho^{1+\alpha}-\frac{1}{R_{1}^{\alpha-1}(\alpha-1)} \rho^{2}$.

The case where $\alpha=1$ is derived in the same manner, by a proper estimation of the $\operatorname{sum} \sum_{i=1}^{\rho R_{1}} \frac{1}{i}$.

Let us point out that the asymptotic for large values of $\rho$ coincide with what is expressed in Lemma 8.5 in 13. In fact for $\alpha>1$ i.e. if $U(r)$ is not in $L^{1}$ around zero, the leading term in the expression presented is of order $\rho^{1+\alpha}$. Moreover, we also observe that for those values of $\alpha$ that makes $U(r)$ integrable around zero $(\alpha<1)$ the leading order of growth is again $\rho^{2}$. This result is in agreement with what has been proven in 13 for integrable potentials and it also confirms the order of growth derived by Oelschläger in $[9]$.

In summary our claim is that: for large values of $\rho$ the asymptotic of $P_{V}(\rho)$ obeys to the following rule:

$$
\begin{cases}\rho^{1+\alpha} & \text { if } \alpha>1 \\ \rho^{2} & \text { if } \alpha<1 \\ \rho^{2} \log (\rho) & \text { if } \alpha=1\end{cases}
$$




\begin{tabular}{|c|c|c|c|c|}
\hline & \multicolumn{2}{|r|}{$\rho \approx 0.2$} & \multicolumn{2}{|r|}{$\rho \approx 6$} \\
\hline & $V(x)=\left(\frac{1}{\alpha|x|^{\alpha}}-C\right)$ & $V(x)=\left(\frac{1}{\alpha|x|^{\alpha}}-C\right) \mathbb{1}_{|x| \leq R_{1}}$ & $V(x)=\left(\frac{1}{\alpha|x|^{\alpha}}-C\right)$ & $V(x)=\left(\frac{1}{\alpha|x|^{\alpha}}-C\right) \mathbb{1}_{|x| \leq R_{1}}$ \\
\hline$\alpha=0.5$ & 1.001 & 1.013 & 2.04 & 1.48 \\
\hline$\alpha=1$ & 1.03 & 1.025 & 2.13 & 2.03 \\
\hline$\alpha=2$ & 1.027 & 1.017 & 3.004 & 3.004 \\
\hline$\alpha=3$ & 1.025 & 1.023 & 4.007 & 4.007 \\
\hline$\alpha=4$ & 1.009 & 1.011 & 5.003 & 5.003 \\
\hline
\end{tabular}

Table 1: Leading exponent for the function $P_{V}(\rho)$ for various potential, for different values of $\alpha$ and $\rho$ in dimension $d=1$. Note that there are some discrepancies in the case $\alpha<1$ and $\rho \approx 6$ since one recover the theoretical behavior proved in [13] only in the case where the potential is not compactly supported.

For small values of $\rho$ the behavior instead is fully linear in all the analyzed cases. The asymptotic for small and large $\rho$ is confirmed by numerical simulations. In fact we computed the function $P_{V}(\rho)$ for different values of $\rho$, very small for the small density asymptotic and very large for the high density. Afterwards we computed an approximation of the growth exponent by measuring the slope of the function $P_{V}(\rho)$ in $\log \log$-scale. All the results are presented in Table 1 .

Moreover we also compare directly the values obtained by the function $P_{V}(\rho)$ computed by numerical simulations and that obtained by the formula of Claim 1. This comparison is highlighted in Figure 2. As we can see the global regime is captured nicely by the function captured by heuristics. We also compare the plots in $\log \log$ scale, in order to have a better insight on what is the leading term for each values of $\rho$. In both plots the function proposed in Claim 1 has been multiplied by a coefficient 1.6 to obtain a better approximation. This shows that the conjecture proposed in Claim 1, even if correct in terms of the growth exponent, but still needs a small refining in term of multiplicative constants.

In the multidimensional case the situation is more complicate, therefore we only sketch the main computation to derive the leading order term for high values of $\rho$. Assume dimension $d$ and assume $N=\rho L^{d}$ to be such that $N^{1 / d}$ is an integer. In the multidimensional case is less clear how to compute equilibrium points $\left(\bar{x}^{i, N}\right)_{i=1, \ldots, N}$. Hence for simplicity we assume that points $\bar{x}^{i, N}$ ) are arranged on a uniform grid $\mathbb{Z}^{d} / L \mathbb{Z}^{d}$ embedded inside $\mathbb{T}_{L}^{d}$ :

$$
\bar{x}^{i, N}=\left(\frac{i_{1}}{\rho^{1 / d}}, \ldots, \frac{i_{d}}{\rho^{1 / d}}\right) \quad i_{k}=1, \ldots, N^{1 / d} .
$$

With this choice the distance of the $i$-th particle and the particle positioned in the origin is

$$
\frac{\left|\left(i_{1}, \ldots, i_{d}\right)\right|}{\rho^{1 / d}}
$$




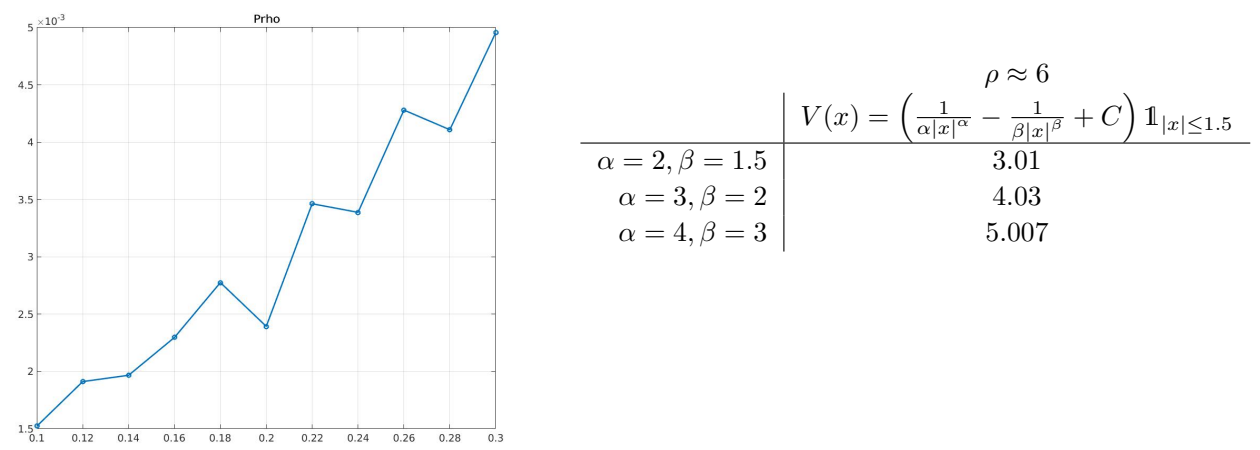

Figure 3: Left: Plots of the function $P_{V}(\rho)$ in the attractive-repulsive case computed numerically, for small values of $\rho$. The estimated exponent by $\log \log$ scale is 1.0265 , confirming the linearity expressed in (4.8). The slope estimated is around 0.0175 which does not coincide with the vale expected $\sigma^{2} / 2=0.005$. Right: Leading exponent of the function $P_{V}(\rho)$ in the attractive-repulsive case for different values of $\alpha, \beta$ and large $\rho$ in dimension $d=1$.

By formula 4.1) we obtain

$$
\begin{aligned}
& -\frac{1}{L^{d}} \sum_{i=1}^{N_{\rho, L}} \sum_{j=1}^{N_{\rho, L}} V^{\prime}\left(\left|\bar{x}^{i, N}-\bar{x}^{j, N}\right|\right)\left|\bar{x}^{i, N}-\bar{x}^{j, N}\right|=-\rho \sum_{i=1}^{\rho L^{d}} V^{\prime}\left(\left|\bar{x}^{i, N}\right|\right)\left|\bar{x}^{i, N}\right| \\
= & \rho \sum_{i=1}^{\rho L^{d}} \frac{\rho^{\alpha / d}}{\left|\left(i_{1}, \ldots, i_{d}\right)\right|^{\alpha}} \mathbb{1}_{\left|\left(i_{1}, \ldots, i_{d}\right)\right| \leq R_{1} \rho^{1 / d}}=\rho^{1+\alpha / d} \sum_{i=1}^{\rho L^{d}} \frac{1}{\left|\left(i_{1}, \ldots, i_{d}\right)\right|^{\alpha}} \mathbb{1}_{\left|\left(i_{1}, \ldots, i_{d}\right)\right| \leq R_{1} \rho^{1 / d} .}
\end{aligned}
$$

Carrying out an explicit computation here is more difficult. However in the case where $R_{1}=+\infty$ and $\alpha>d$, since the series

$$
\sum_{i=1}^{\infty} \frac{1}{\left|\left(i_{1}, \ldots, i_{d}\right)\right|^{\alpha}}<\infty
$$

we understand that the leading term in the expression of $P_{V}(\rho)$ is $\rho^{1+\alpha / d}$. This intuition is confirmed by the values computed numerically in the case $d=2$, Table 2

\subsection{Attractive-repulsive potential}

We now consider the attractive-repulsive case, see Potential given by 4.5. In this case the potential $U(x)$ change the sign, see Figure 4. Let us now start to discuss equilibrium configurations for particles 4.3 in this case. Again we propose the argument in dimension $d=1$ for a matter of simplicity. In this case the minimum of the potential $U$ is obtained in the values $R_{0}$. Therefore particles have the tendency to align at a distance of $R_{0}$ one to each other. Moreover we will always analyze 


\begin{tabular}{l|cc|} 
& \multicolumn{2}{|c|}{$\rho \approx 6$} \\
& $V(x)=\left(\frac{1}{|x|^{\alpha}}-C\right)$ & $V(x)=\left(\frac{1}{|x|^{\alpha}}-C\right) \mathbb{1}_{|x| \leq R_{1}}$ \\
\hline$\alpha=1$ & 2.031 & 1.421 \\
$\alpha=2$ & 2.11 & 2.04 \\
$\alpha=3$ & 2.503 & 2.511 \\
$\alpha=4$ & 3.04 & 3.07 \\
$\alpha=5$ & 3.54 & 3.55
\end{tabular}

Table 2: Leading exponent for the function $P_{V}(\rho)$ for various potential, for different values of $\alpha$ for large $\rho$ in dimension $d=2$.

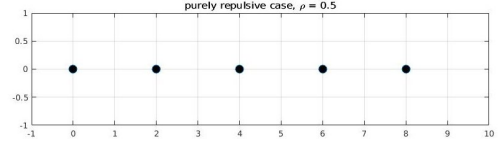

purely repulisive case,,-1

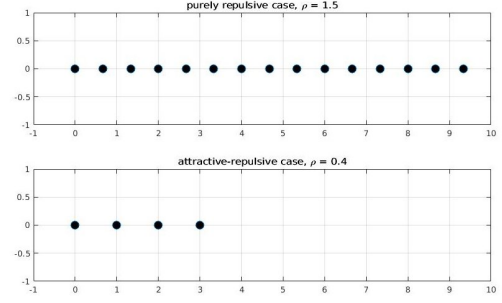

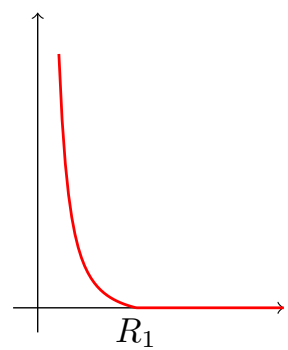

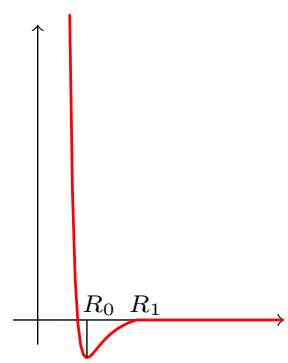

Figure 4: Left: Equilibrium configurations in dimension $d=1$. The distances between consecutive particles is of order $1 / \rho$ in the repulsive case, and of order $R_{1}$ in the attractive case for $\rho<1 / R_{1}$. Right: the potential $V(x)$ in the purely repulsive case (top) and attractive repulsive case (bottom).

the case where $R_{1}<2 R_{0}$. This conditions specifies the fact that, if a particle is located at distance $R_{0}$ from its two neighbors, then it interacts only with the two of them, since other particles are outside the support of the potential $V(x)$. If we think particles as cells of radius $R_{0} / 2$, we are describing the phenomenon of cellular adhesion, indeed attraction occurs only when cells are in contact, namely when they are at distance smaller then $2 R_{0}$. This assumption creates an uniform structure: the equilibrium configuration consists of particles collocated at equal distance $R_{0}$, see Figure 4 It is worth noting that this configuration is not uniformly spread in the whole torus. In fact for low values of $\rho$ particles will tend to form a cluster that occupies only a portion of the available space. When the density is higher, $\rho>1 / R_{0}$, particles are compressed to be closer than $R_{0}$ one from the other, and we recover the 
uniform distribution at distance $1 / \rho$ as in the purely repulsive case. This intuition is confirmed by numerical simulations where we had the system start at a random initial condition and let it evolve towards its stationary configuration. Summarizing we imagine the equilibrium configuration in the attractive-repulsive case to be

$$
\begin{cases}\text { equally spaced particles at distance } R_{0} & \text { if } \rho<1 / R_{0} \\ \text { equally spaced particles at distance } 1 / \rho & \text { if } \rho \geq 1 / R_{0}\end{cases}
$$

Notice that the situation here is different from the purely repulsive case particles will always place at a distance $1 / \rho$ for every value of $\rho$. We now present a claim for

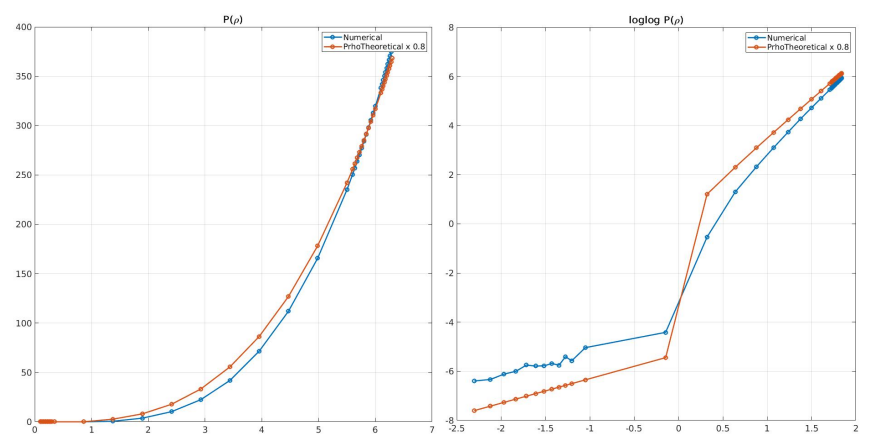

Figure 5: Attractive repulsive case with compact support, $\alpha=2, \beta=1.5, R_{1}=1.5$ in dimension $d=1$. Comparison between the function $P_{V}(\rho)$ obtained by numerical simulations and by that obtained by heuristics. Left: comparison between functions $P_{V}(\rho)$ in natural scale. Right: Comparison in $\log \log$ scale. The theoretical function computed as in Claim 1 has been rescaled by 0.8 .

the function $P_{V}(\rho)$ in the attractive-repulsive case. For simplicity we will present only the case where $\alpha>\beta>1$.

Claim 2. In dimension $d=1$, if the potential $U$ is given by 4.5 we claim that

$$
P_{V}(\rho) \approx \begin{cases}\sigma^{2} \rho+O(\rho) & \rho<1 / R_{0}, \\ \sigma^{2} \rho+\frac{\alpha}{\alpha-1} \rho^{1+\alpha}-\frac{\beta}{\beta-1} \rho^{1+\beta}-\left(\frac{1}{R_{1}^{\alpha-1}(\alpha-1)}-\frac{1}{R_{1}^{\beta-1}(\beta-1)}\right) \rho^{2} & \rho \geq 1 / R_{0}\end{cases}
$$

By repeating the same argument proposed on the repulsive case, we get as approximation for $P_{V}(\rho)$ :

$$
P_{V}^{h}(\rho) \approx \begin{cases}\sigma^{2} \rho & \rho<1 / R_{0} \\ \sigma^{2} \rho+\frac{\alpha}{\alpha-1} \rho^{1+\alpha}-\frac{\beta}{\beta-1} \rho^{1+\beta}-\left(\frac{1}{R_{1}^{\alpha-1}(\alpha-1)}-\frac{1}{R_{1}^{\beta-1}(\beta-1)}\right) \rho^{2} & \rho \geq 1 / R_{0}\end{cases}
$$

In this case numerical simulations and the heuristic result show some tiny discrepancies. We observe some difference regarding the multiplicative constant but no 
differences are observed on the side of growth exponent. First we analyze the behavior for small $\rho$, Figure 3 . Here we see that, while the growth is indeed linear, the slope of the curve does not coincide with the value $\sigma^{2} / 2$ which has been conjectured in 4.8). This shows that in the attractive repulsive case there could be some additional contribution of the order $O(\rho)$ that has not yet been identified. Moreover we also analyze, via the slope of the function $P_{V}(\rho)$ in $\log \log$ scale, the leading exponent for large values of $\rho$. The results are collected again on Figure 3. Here we confirm that the leading asymptotic exponent is given by $1+\alpha$ as in the purely repulsive case. In summary we can conclude that up to some correction term, not clearly identified, but of order $O(\rho)$ the function proposed by the heuristic argument catches the global behavior of $P_{V}(\rho)$.

\section{Bibliography}

1. Nicola J Armstrong, Kevin J Painter, and Jonathan A Sherratt. A continuum approach to modelling cell-cell adhesion. Journal of theoretical biology, 243(1):98-113, 2006.

2. Maria Bruna and S Jonathan Chapman. Excluded-volume effects in the diffusion of hard spheres. Physical Review E, 85(1):011103, 2012.

3. Andreas Buttenschoen, Thomas Hillen, Alf Gerisch, and Kevin J Painter. A space-jump derivation for non-local models of cell-cell adhesion and non-local chemotaxis. Journal of mathematical biology, 76(1-2):429-456, 2018.

4. Franco Flandoli and Marta Leocata. A particle system approach to aggregation phenomena. Journal of Applied Probability, 56(1):282-306, 2019.

5. Nir Gavish, Pierre Nyquist, and Mark Peletier. Large deviations and gradient flows for the brownian one-dimensional hard-rod system. arXiv preprint arXiv:1909.02054, 2019.

6. Claude Kipnis and Claudio Landim. Scaling limits of interacting particle systems, volume 320. Springer Science \& Business Media, 1998.

7. Karl Oelschläger. A law of large numbers for moderately interacting diffusion processes. Zeitschrift für Wahrscheinlichkeitstheorie und verwandte Gebiete, 69(2):279-322, 1985.

8. Karl Oelschläger. On the derivation of reaction-diffusion equations as limit dynamics of systems of moderately interacting stochastic processes. Probability Theory and Related Fields, 82(4):565-586, 1989.

9. Karl Oelschläger. Large systems of interacting particles and the porous medium equation. Journal of differential equations, 88(2):294-346, 1990.

10. Hermann Rost. Diffusion de sphéres dures dans la droite réelle: comportement macroscopique et équilibre local. In Séminaire de Probabilités XVIII 1982/83, 127-143. Springer, 1984.

11. Nicolas Rougerie. De finetti theorems, mean-field limits and bose-einstein condensation. arXiv preprint arXiv:1506.05263, 2015.

12. Steven Schochet. The weak vorticity formulation of the 2-d euler equations and concentration-cancellation. Communications in partial differential equations, 20(56):1077-1104, 1995.

13. Kôhei Uchiyama. Pressure in classical statistical mechanics and interacting brownian particles in multi-dimensions. In Annales Henri Poincaré, volume 1, 1159-1202. Springer, 2000.

14. SRS Varadhan. Scaling limits for interacting diffusions. Communications in mathematical physics, 135(2):313-353, 1991. 